\title{
LETTERS
}

\section{The authors respond to: "Case did not consider all potential causes of chilblains"}

We thank Dr. Dewar ${ }^{1}$ for her interest in our CMAJ article. ${ }^{2}$ We considered a broad workup for contributory and aggravating causes and conducted a complete assessment during the clinical encounter. However, this could not all be captured within the article's word limit. For clarification, we provide further details.

During the early months of the coronavirus disease 2019 (COVID-19) pandemic, our patient was assessed in-person in the emergency department, enabling a thorough history and a complete physical examination. Our patient had no history of nicotine or psychostimulant consumption. In addition, her review of systems was negative, including for those of autoimmune disorders and malignant disease (i.e., no fever, night sweats, weight changes, oral or genital ulcerations, photosensitivity, arthritis, arthralgias, morning stiffness, myalgias, muscle weakness, sicca symptoms or other cutaneous eruptions). The patient also did not have a family history of known autoimmune conditions. Other than the acral lesions described in our article, her physical examination was unremarkable. In particular, she had a normal body habitus without clinical signs of anorexia nervosa, no other cutaneous findings such as oral ulcerations or photo-distributed lesions, and normal nail capillaroscopy. We did not pursue a musculoskeletal examination given the lack of symptoms.
We completed a thorough panel of investigations to rule out systemic causes of chilblains. We did not conduct imaging investigations given the absence of respiratory symptoms before, during and after clinical encounter, and because chest imaging is not recommended to diagnose COVID-19 in patients with mild features. ${ }^{3}$ In addition, imaging would not have altered the cutaneous management.

We agree that a skin biopsy is not always required for diagnosis of chilblains because the findings are not pathognomonic but rather supportive of a clinical diagnosis. Our patient presented near the beginning of the pandemic when little was known about severe acute respiratory syndrome coronavirus 2 (SARS-CoV-2)related chilblains. There have been reports of classic chilblains histology associated with SARS-CoV-2 infection, as well as superficial and deep lichenoid lymphocytic changes with basal vacuolar changes; ${ }^{4,5}$ microthrombi have also been described..$^{6,7} \mathrm{~A}$ biopsy was completed to assess for microthrombi and to rule out vaso-occlusive pathology.

Idiopathic chilblains remain a diagnosis of exclusion. Patients presenting with chilblains should be appropriately examined and investigated to rule out secondary systemic causes. Our patient's thorough history, physical examination and subsequent workup did not support any underlying disorder. Chilblains related to SARS-CoV-2 was favoured because of negative secondary workup, absence of cool/ damp exposure and positive results for serology testing. The understanding of
SARS-CoV-2-related chilblains and its pathogenesis will continue to evolve in the coming months.

\section{Malika A. Ladha MD}

Resident physician, Division of

Dermatology, Department of Medicine, University of Calgary, Calgary, Alta.

\section{Elaine C. Dupuis MD}

Dermatologist, Division of Dermatology, Department of Medicine, University of Calgary, Calgary, Alta.

Cite as: CMAJ 2020 September 8;192: E1047. doi: $10.1503 / \mathrm{cmaj} .76386$

\section{References}

1. Dewar C. Case did not consider all potential causes of chilblains [letter]. CMAJ 2020;192:E1046.

2. Ladha MA, Dupuis EC. SARS-CoV-2-related chilblains. CMAJ 2020;192:E804.

3. Dennie C, Hague C, Lim RS, et al. Canadian Society of Thoracic Radiology/Canadian Association of Radiologists consensus statement regarding chest imaging in suspected and confirmed COVID-19. Can Assoc Radiol J 2020 May 8 [Epub ahead of print]. doi:10.1177/0846537120924606.

4. Kolivras A, Dehavay F, Delplace D, et al. Coronavirus (COVID-19) infection-induced chilblains: a case report with histopathological findings. JAAD Case Rep 2020;6:489-92.

5. Cordoro KM, Reynolds SD, Wattier R, et al. Clustered cases of acral perniosis: clinical features, histopathology and relationship to COVID-19. Pediatr Dermatol 2020;37:419-23.

6. Andina D, Noguera-Morel L, Bascuas-Arribas M, et al. Chilblains in children in the setting of COVID-19 pandemic. Pediatr Dermatol 2020;37: 406-11.

7. de Masson A, Bouaziz JD, Sulimovic L, et al. Chilblains are a common cutaneous finding during the COVID-19 pandemic: a retrospective nationwide study from France. J Am Acad Dermatol 2020;83:667-70.

Competing interests: None declared. 